\title{
The Deep Inferior Epigastric Perforator and Pedicled Transverse Rectus Abdominis Myocutaneous Flap in Breast Reconstruction: A Comparative Study
}

\author{
Shane $\operatorname{Tan}^{1}$, Jane Lim², Jacklyn Yek ${ }^{1}$, Wei Chen Ong' 2 , Chor Hoong Hing 2 , Thiam Chye Lim² \\ ${ }^{1}$ National University Health System, National University Hospital, Singapre; ${ }^{2}$ Division of Plastic, Reconstructive and Aesthetic Surgery, \\ Department of Surgery, National University Hospital, University of Singapore, Singapore, Singapore
}

Background Our objective was to compare the complication rates of two common breast reconstruction techniques performed at our hospital and the cost-effectiveness for each test group.

Methods All patients who underwent deep inferior epigastric perforator (DIEP) flap and transverse rectus abdominis myocutaneous (TRAM) flap by the same surgeon were selected and matched according to age and mastectomy with or without axillary clearance. Patients from each resultant group were selected, with the patients matched chronologically. The remainder were matched for by co-morbidities. Sixteen patients who underwent immediate breast reconstruction with pedicled TRAM flaps and 16 patients with DIEP flaps from 1999 to 2006 were accrued. The average total hospitalisation cost, length of hospitalisation, and complications in the 2 year duration after surgery for each group were compared.

Results Complications arising from both the pedicled TRAM flaps and DIEP flaps included fat necrosis (TRAM, 3/16; DIEP, 4/16) and other minor complications (TRAM, 3/16; DIEP, 1/16). The mean hospital stay was 7.13 days (range, 4 to 12 days) for the pedicled TRAM group and 7.56 (range, 5 to 10 days) for the DIEP group. Neither the difference in complication rates nor in hospital stay duration were statistically significant. The total hospitalisation cost for the DIEP group was significantly higher than that of the pedicled TRAM group $(P<0.001)$.

Conclusions Based on our study, the pedicled TRAM flap remains a cost-effective technique in breast reconstruction when compared to the newer, more expensive and tedious DIEP flap.

Keywords Perforator flap / Surgical flap / Mammoplasty / Complications
Correspondence: Jane Lim Division of Plastic, Reconstructive and Aesthetic Surgery, Department of Surgery, National University Hospital, Lower Kent Ridge Road, Singapore 119074, Singapore

Tel: $+65-67722022$

Fax: +65-67722336

E-mail: cfslimj@nus.edu.sg

The authors would like to thank Dr. Mikael Hartman, Assistant Professor MD, PhD; Department of Epidemiology and Public Health, National University of Singapore; Department of Surgery, Yong Loo Lin School of Medicine, National University of Singapore, for his advice on statistical issues.

This study was done in accordance with National Healthcare Group ethics guidelines (DSRB D/09/056).

This article was presented as a poster at the BMJ International Forum on Quality \& Safety in Healthcare 2012 on April 16-19, 2012 in Paris.

No potential conflict of interest relevant to this article was reported.

Received: 14 Feb 2013 • Revised: 27 Mar 2013 • Accepted: 4 Apr 2013

pISSN: 2234-6163 • elSSN: 2234-6171 • http://dx.doi.org/10.5999/aps.2013.40.3.187• Arch Plast Surg 2013;40:187-191

\section{INTRODUCTION}

Many techniques for autologous breast reconstruction have evolved [1] since the latissimus dorsi flap [2] and the transverse rectus abdominis myocutaneous (TRAM) flap [3] were introduced in the 1970s and 1982, respectively. The use of microvascular techniques and perforator flaps [4] soon evolved to minimize donor site morbidities. The method of choice should

Copyright (C) 2013 The Korean Society of Plastic and Reconstructive Surgeons

This is an Open Access article distributed under the terms of the Creative Commons Attribution Non-Commercial License (http://creativecommons.org/

licenses/by-nc/3.0/) which permits unrestricted non-commercial use, distribution, and reproduction in any medium, provided the original work is properly cited.

www.e-aps.org 
be safe, reliable, and should result in little or no donor-site morbidity. As techniques become more sophisticated, it is timely to assess the cost effectiveness of these reconstruction approaches.

In autologous breast reconstruction, the abundant adipose tissue present in the lower abdomen of most women is often used and has become the most popular donor tissue for breast reconstruction [5]. Refinements include the use of microvascular techniques, the muscle sparing TRAM flap, and the deep inferior epigastric perforator (DIEP) flap.

Of the various existing techniques available, the pedicled TRAM and DIEP flaps have shown to be the preferred forms of breast reconstructive techniques [6]. Perforator flaps such as the DIEP flap are associated with fewer donor site deficits. There are many proponents of DIEP flap reconstruction and in recent years, there has been an increasing preference for the DIEP flap in autologous breast reconstruction [5-8]. To date, few studies have compared the cost effectiveness of the two commonly used reconstructive techniques. The aim of this paper is to evaluate the two procedures to determine the cost effectiveness with regard to the total cost and postoperative complications.

The determination of cost difference would be of particular benefit in countries such as Singapore, as healthcare costs (depending on the class one selects, and hence the cost born by the payee and level of privacy in the ward) could be lowered for both the patient as well as the government, which subsidises based on ward class selection, allowing for redirection of cash reserves.

\section{METHODS}

A retrospective analysis of the patients at our institution who underwent breast reconstruction with either a pedicled TRAM flap or DIEP flap from 1999 to 2006 was performed. The choice of procedure was selected at two points: first, selected randomly prior to the operation; then, intraoperatively depending on the vascular status of the patient. A patient initially selected at random for a DIEP procedure would then be assessed intra-operatively for good microvascular status before proceeding with the DIEP reconstruction; poor candidates who were deemed unsuitable were subsequently reconstructed with the pedicled TRAM.

Selection criteria for the study included: women who underwent unilateral mastectomy with or without axillary clearance and immediate post-mastectomy breast reconstruction. Patients who had bilateral mastectomy and reconstruction, delayed reconstruction, chest wall resection, or patients who underwent revision surgery to the contralateral breast or ipsilateral breast after reconstruction were excluded from the study. The test sub- jects were then matched for age, mastectomy without axillary clearance, mastectomy with axillary clearance, and the comorbidities elaborated below.

Patients selected by the above method were matched by age in intervals of five years ( 31 to 35 years, 36 to 40 years, 41 to 45 years, etc.), comorbidities, and mastectomy procedure. Comorbidities that were considered included diabetes mellitus, a history of vascular insufficiency, smoking and body mass index $>30$. This was done to minimise the effect of confounding factors in the study, although some studies suggest that complication rates amongst patients undergoing autologous abdominal flap reconstruction, whether DIEP or pedicled TRAM, are associated with an overall low morbidity rate regardless of associated comorbidities [9]. From an initial pool of 50 pedicled TRAM and 30 DIEP cases, thirty-two patients were matched: sixteen patients with DIEP flaps and 16 patients with pedicled TRAM flaps. The matched patients' ages ranged from 31 to 50 years, with an average age of 43.8 years.

We looked at postoperative complications up to two years postoperation, the average length of stay and total hospitalization cost for each of the patients.

Immediate post-reconstructive complications were categorized into major and minor categories. Major complications consisted of total flap loss, fat necrosis and abdominal hernias; minor complications included wound dehiscence, infection (donor or recipient site), postoperative haematoma requiring evacuation and seroma formation (Table 1).

Total flap loss was defined as complete necrosis of the skin and fat; fat necrosis was defined as loss of a portion of the adipose components. To determine the total hospitalisation cost of the procedure for each patient, the following were examined and extracted for comparison: operative fees (surgical, facility, anaesthetic), length of ward stay, daily treatment fees, daily pharmaceutical fees and cost of implants (prolene mesh). Facility fees included usage of operating room and surgical consumables e.g., sutures, microscope and instruments. Length of stay was defined as the number of days from the day of admission to that of discharge.

Statistical analysis was performed using two-tailed t-tests for cost differences and average length of hospitalisation, and Fish-

Table 1. Classification of post-reconstructive complications

\begin{tabular}{|ll|}
\hline Major complications & \multicolumn{1}{c|}{ Minor complications } \\
\hline Total flap loss & Seroma formation \\
Fat necrosis & Wound dehiscence \\
$\begin{array}{l}\text { Abdominal bulge requiring surgical } \\
\text { repair }\end{array}$ & $\begin{array}{c}\text { Postoperative haemorrhage requiring } \\
\text { evacuation } \\
\end{array}$ \\
& Infection (mesh/donor site) \\
\hline
\end{tabular}


Table 2. Post-reconstructive complications in the pedicled TRAM and DIEP groups

\begin{tabular}{|c|c|c|}
\hline & $\begin{array}{c}\text { Major } \\
\text { complications }\end{array}$ & $\begin{array}{c}\text { Minor } \\
\text { complications }\end{array}$ \\
\hline Pedicled TRAM $(n=16)$ & Fat necrosis (3) & $\begin{array}{l}\text { Donor site infection (1) } \\
\text { Wound dehiscence (1) } \\
\text { Postoperative haemorrhage } \\
\text { requiring evacuation (1) }\end{array}$ \\
\hline Total & 3 & 3 \\
\hline $\operatorname{DIEP}(n=16)$ & Fat necrosis (4) & $\begin{array}{l}\text { Postoperative haemorrhage } \\
\text { requiring evacuation (1) }\end{array}$ \\
\hline Total & 4 & 1 \\
\hline P-value $e^{a)}$ & $>0.05$ & 0.599 \\
\hline
\end{tabular}

er's exact test for differences in complication rates. A P-value of less than 0.05 was considered to be statistically significant.

\section{RESULTS}

\section{Complication rates}

Three of 16 pedicled of our pedicled TRAM flap patients had major complications (fat necrosis) and 3/16 had minor complications (one wound dehiscence, one haematoma requiring evacuation, one donor site infection).

Four of 16 patients of the patients who underwent DIEP flap reconstruction had major complications (fat necrosis) and $1 / 16$ had minor complications (haematoma requiring evacuation). The results are depicted in Table 2.

These differences were not statistically significant $(\mathrm{P}>0.05)$.

\section{Cost of operation}

Patients undergoing breast reconstruction with the pedicled TRAM flap had an average total hospitalization cost of SGD 8,300.51 (6,685.33 USD), while patients who underwent DIEP flap reconstruction post-mastectomy had an average total cost of SGD 11,009.38 (8,864.67 USD). The cost difference between the two was statistically significant $\mathrm{P}<0.001$.

\section{Average length of stay}

The pedicled TRAM group had an average stay of 7.13 days (ranging between 4 and 12 days) and the DIEP group had an average stay of 7.56 days (ranging between 5 and 10 days). The difference in the length of time stayed between the two was not statistically significant, defined as $\mathrm{P}>0.05(\mathrm{P}=0.489)$.

\section{DISCUSSION}

Reconstruction of the breast, considering the lack of association with increased morbidity or mortality with regard to surgical or oncologic outcome [10] and its positive psychological effect on patients post-mastectomy [11], is proving to play a more integral role in the management of breast cancer patients.

Breast reconstruction can be performed with autologous tissue, implants, or both. Autologous techniques in breast reconstruction include pedicled flaps (TRAM and latissimus dorsi flaps) and free tissue transfer (TRAM flap, DIEP flap, anterior lateral thigh flap, superior gluteal artery perforator flap). The purpose of the perforator flap is to allow the preservation of muscles and vital structures at the donor site. The DIEP flap is one such flap, where the anterior abdominal wall is kept intact with the preservation of the rectus muscle, its nerve supply and the rectus sheath. While there has been an increasing preference for the DIEP flap in breast reconstruction [5], comparing the DIEP and the pedicled TRAM has not drawn much attention in the literature. Our hypothesis is that, while the use of complicated microsurgical techniques in DIEP flap surgery increases the cost of the procedure [12-14], the decreased donor site morbidity would presumably imply less postoperative pain and hence less use of analgesics and a shorter hospital stay. Whether this translates into a smaller total cost during hospitalization is not easily determined.

The DIEP flap, first described by Koshima and Soeda [7] in 1989 and subsequently popularised by by Allen and Treece [8], and Blondeel [6] preserves the rectus abdominis muscle, its sheath and its segmental nerve supply [15]. This was found to decrease post-reconstructive donor site complications such as hernias, unsightly bulges and asymmetry, and to increase abdominal flexion strength when compared to free and musclesparing TRAM flaps $[16,17]$. A recent study by Momoh et al. [18] of 346 patients from 1999 to 2006 that examined the complications and patient satisfaction from both pedicled TRAM flaps and DIEP flaps showed that the DIEP flap had significantly less donor site morbidity (hernias and abdominal bulges) and greater general satisfaction. Recipient site complications and aesthetic satisfaction were not significantly different [18]. Similarly, Man et al. [19] have shown that DIEP flaps result in fewer donor site complications, though flap-related complications are higher. Patients with DIEP flap reconstruction have also been shown to experience less pain [20], with resultantly quicker recovery and discharge from hospital, and hence a shorter hospital stay with lower costs incurred [21] compared to reconstruction with free TRAM flaps. However, an interesting recent case report has shown the occurrence of epigastric hernias in 3 patients following DIEP flap breast reconstruction [22]

Comparing patient satisfaction, Yueh et al. [23] have shown that when comparing the two reconstructive techniques, nei- 
ther general nor aesthetic satisfaction were statistically different after logistical regression. While no study has compared the frequency of post-reconstructive aesthetic refinements to the breast between the two procedures, Enajat et al. [24] have shown that overall, there was an average of 1.06 additional interventions for every patient after primary reconstructive surgery with the DIEP flap. Secondary interventions included nipple reconstruction, nipple-areola complex tattooing, dog-ear correction, liposuction, lipofilling, scar revision, mastopexy and reduction mammaplasty [24]. However, none had an objective measure to compare the aesthetic result of the completed breast resulting from either procedure either in the short or long term.

The pedicled TRAM flap is a shorter operation, does not use microvascular techniques and the entire rectus muscle and all or part of the rectus sheath is sacrificed, with resulting donor site complications as mentioned above. However, other studies suggest that the inclusion of prolene mesh at the abdominal donor site in pedicled TRAM flap reconstruction allows for the attainment of abdominal complication rates similar to that of DIEP flap reconstruction [25]. This seems to suggest that the pedicled TRAM flap, with the proper use of mesh implants, might still prove to be a reliable technique in breast reconstruction despite the development of newer techniques. This may prove the pedicled TRAM flap to be equally applicable today despite the development of perforator flaps like the DIEP flap.

Our study produced the following results: the pedicled TRAM group had a greater complication rate in terms of minor complications while the DIEP group had more major complications, although neither were significantly different. The overall immediate cost to the patient was, however, significantly different, with DIEP flap patients paying an average of SGD 2708.87 more (TRAM, SGD 8300.51; DIEP, SGD 11009.38).

The DIEP group had a higher total cost due to the following: 1) Use of microsurgical techniques and equipment. 2) More costly consumables such as microsutures and heparinized solutions. 3) Longer operating hours (facility, surgeon and anesthetic fees).

We found no evidence of immediate additional benefit of DIEP flap reconstruction over a pedicled TRAM flap reconstruction, although there was also no evidence of increased morbidity with the DIEP flap.

As such, the pedicled TRAM flap is still be a highly reliable technique in breast reconstruction despite the development of more complex and purportedly more beneficial procedures such as the DIEP flap. This is, at least, with regard to the factors considered above. There is a need for long term monitoring of patient satisfaction as well as objective monitoring of the longterm evolution of the reconstructed breast and any additional cost incurred that might follow in addressing long-term complications.

In conclusion, despite the total hospitalization cost of performing a DIEP flap breast reconstruction being higher than reconstruction with a pedicled TRAM flap $(\mathrm{P}<0.001)$, differences in average length of hospitalisation and complication rates were not statistically significant. As such, the extra cost of performing a more sophisticated reconstruction with the DIEP flap is not supported here. The pedicled TRAM flap remains a cost effective reconstructive technique in breast reconstruction.

A longer follow up, objective assessment of the aesthetic outcome and consideration of costs and complications of subsequent corrective surgery and quality of life in these two groups would produce a more accurate cost-benefit analysis of these two methods of reconstruction.

\section{REFERENCES}

1. Petit J, Rietjens M, Garusi C. Breast reconstructive techniques in cancer patients: which ones, when to apply, which immediate and long term risks? Crit Rev Oncol Hematol 2001; 38:231-9.

2. Tansini I. Sopra il mio nuovo processo di amputazione della mammella. Gazz Med Ital 1906;57:141.

3. Hartrampf CR, Scheflan M, Black PW. Breast reconstruction with a transverse abdominal island flap. Plast Reconstr Surg 1982;69:216-25.

4. Saint-Cyr M, Schaverien MV, Rohrich RJ. Perforator flaps: history, controversies, physiology, anatomy, and use in reconstruction. Plast Reconstr Surg 2009;123:132e-45e.

5. Gurunluoglu R, Gurunluoglu A, Williams SA, et al. Current trends in breast reconstruction: survey of American Society of Plastic Surgeons 2010. Ann Plast Surg 2013;70:103-10.

6. Blondeel PN. One hundred free DIEP flap breast reconstructions: a personal experience. Br J Plast Surg 1999;52: 104-11.

7. Koshima I, Soeda S. Inferior epigastric artery skin flaps without rectus abdominis muscle. Br J Plast Surg 1989;42:645-8.

8. Allen RJ, Treece P. Deep inferior epigastric perforator flap for breast reconstruction. Ann Plast Surg 1994;32:32-8.

9. Seidenstuecker K, Munder B, Mahajan AL, et al. Morbidity of microsurgical breast reconstruction in patients with comorbid conditions. Plast Reconstr Surg 2011;127:1086-92.

10. Rozen WM, Ashton MW, Taylor GI. Defining the role for autologous breast reconstruction after mastectomy: social and oncologic implications. Clin Breast Cancer 2008;8:13442.

11. Wilkins EG, Cederna PS, Lowery JC, et al. Prospective anal- 
ysis of psychosocial outcomes in breast reconstruction: oneyear postoperative results from the Michigan Breast Reconstruction Outcome Study. Plast Reconstr Surg 2000;106: 1014-25.

12. Marchac A, Bosc R, Benjoar MD, et al. A cost analysis of DIEP flap in breast reconstruction. Ann Chir Plast Esthet 2011;56:275-9.

13. Damen TH, Wei W, Mureau MA, et al. Medium-term cost analysis of breast reconstructions in a single Dutch centre: a comparison of implants, implants preceded by tissue expansion, LD transpositions and DIEP flaps. J Plast Reconstr Aesthet Surg 2011;64:1043-53.

14. Thoma A, Veltri K, Khuthaila D, et al. Comparison of the deep inferior epigastric perforator flap and free transverse rectus abdominis myocutaneous flap in postmastectomy reconstruction: a cost-effectiveness analysis. Plast Reconstr Surg 2004;113:1650-61.

15. Lee SJ, Lim J, Tan WT, et al. Changes in the local morphology of the rectus abdominis muscle following the DIEP flap: an ultrasonographic study. Br J Plast Surg 2004;57:398-405.

16. Blondeel N, Vanderstraeten GG, Monstrey SJ, et al. The donor site morbidity of free DIEP flaps and free TRAM flaps for breast reconstruction. Br J Plast Surg 1997;50:322-30.

17. Atisha D, Alderman AK. A systematic review of abdominal wall function following abdominal flaps for postmastectomy breast reconstruction. Ann Plast Surg 2009;63:222-30.

18. Momoh AO, Colakoglu S, Westvik TS, et al. Analysis of complications and patient satisfaction in pedicled transverse rectus abdominis myocutaneous and deep inferior epigastric perforator flap breast reconstruction. Ann Plast Surg 2012; 69:19-23.

19. Man LX, Selber JC, Serletti JM. Abdominal wall following free TRAM or DIEP flap reconstruction: a meta-analysis and critical review. Plast Reconstr Surg 2009;124:752-64.

20. Kroll SS, Sharma S, Koutz C, et al. Postoperative morphine requirements of free TRAM and DIEP flaps. Plast Reconstr Surg 2001;107:338-41.

21. Kaplan JL, Allen RJ. Cost-based comparison between perforator flaps and TRAM flaps for breast reconstruction. Plast Reconstr Surg 2000;105:943-8.

22. Conroy K, Malata CM. Epigastric hernia following DIEP flap breast reconstruction: complication or coincidence? J Plast Reconstr Aesthet Surg 2012;65:387-91.

23. Yueh JH, Slavin SA, Adesiyun T, et al. Patient satisfaction in postmastectomy breast reconstruction: a comparative evaluation of DIEP, TRAM, latissimus flap, and implant techniques. Plast Reconstr Surg 2010;125:1585-95.

24. Enajat M, Smit JM, Rozen WM, et al. Aesthetic refinements and reoperative procedures following 370 consecutive DIEP and SIEA flap breast reconstructions: important considerations for patient consent. Aesthetic Plast Surg 2010;34: 306-12.

25. Mizgala CL, Hartrampf CR Jr, Bennett GK. Abdominal function after pedicled TRAM flap surgery. Clin Plast Surg 1994;21:255-72. 